\title{
Audit of an emergency biochemistry service
}

\author{
W S A Smellie, M J Murphy, P J Galloway, J Hinnie, J McIlroy, F J Dryburgh
}

\begin{abstract}
Aim-To examine a model for the evaluation of appropriateness of testing in an emergency biochemistry laboratory.

Methods-A model was devised in which incoming emergency test requests were categorised as appropriate or inappropriate. Explicit criteria were used to define eight minor categories, which were chosen to reflect accurately current working practice within the hospital and laboratory. Five junior medical staff each undertook a prospective 24 hour assessment, during which time all incoming requests were monitored and categorised according to these criteria. Concordance between monitors was evaluated before and during assessments.

Results-Of 509 requests, 384 (75\%) were appropriate and 125 (25\%) were inappropriate according to the criteria used to define categories. Inappropriate requests fell into three main groups: preoperative samples $(43 \cdot 2 \%(54 / 125)$ of all inappropriate requests), missed routine samples $(33 \cdot 6 \%(42 / 125))$ and accelerated (priority) analyses (16\% (20/125)). Various other reasons accounted for the remaining $7 \cdot 2 \%(9 / 125)$.

Conclusion-This model may be used to obtain valid information about current clinical and laboratory practice. Strategies to reduce the number of inappropriate requests have been identified in order to reserve the emergency service for situations of true need.

(f Clin Pathol 1995;48:1126-1129)
\end{abstract}

Keywords: Audit, appropriateness, emergency laboratory.

Inclusion of measurements of appropriateness may considerably influence future medical practice both in the diagnostic techniques used and in the interventions performed. ${ }^{1}$ This should allow resources to be targeted more effectively and may potentially help to minimise less useful or unsuitable management.

The term appropriateness may be defined as the suitability of the use of a resource for the purposes for which it is designed. Within the field of medical management it may be applied to diagnostic testing in order to examine the use of resources within a service. In a clinical biochemistry laboratory, the emergency service may be used, firstly, for the urgent investigation of acutely ill patients and, secondly, to assist in the decision to admit patients to hospital. Often emergency tests are carried out to expedite the administration of acute admissions, even though the decision to admit has been made on other grounds. For the purposes of the present study, this too is considered an appropriate use of the service. It is important, however, that the emergency service be reserved for situations of true need both to maintain the quality of acute medical care and also to limit the costs of a resource intensive, single sample service. This may best be achieved by obtaining valid information of current practice which may then be used in discussion with ward based clinical staff to target resources. We have examined a model for the evaluation of appropriateness of test requesting in our emergency biochemistry laboratory.

Requests which do not meet the above criteria for an emergency biochemistry service may be considered inappropriate. This does not imply incorrect or poor clinical practice; the inappropriate label may, in fact, be as much a function of laboratory practice as of clinical practice. However, it may be useful to define a group of requests for which an alternative, less costly, mechanism for analysis is desirable. To do this, the laboratory must first classify and quantify those requests which do not fit its criteria for an appropriate test. Improvements in the organisation of a laboratory service have been made by examining requesting patterns of service users in this way. ${ }^{2}$

Using the criteria outlined above, we examined the appropriateness of requests for emergency biochemistry analyses at Glasgow Royal Infirmary over five 24 hour periods and categorised the requests into two major and eight minor categories. Here, we present a description of the method used to evaluate appropriateness, a breakdown of the different categories of requests received, and a discussion of the implications of these findings for clinical and/or laboratory practice within the hospital.

\section{Methods}

This evaluation was performed in June 1994 by five junior medical staff at the Institute of Biochemistry, Glasgow Royal Infirmary. Each undertook a prospective assessment over a (different) weekday 24 hour period starting at 0900 hours. All emergency requests received during this period were monitored. Each request was categorised on a study form (figure). Categorisation was made either while telephoning the results directly to the requesting clinician or ward, or by contacting the ward retrospectively if results had already been released. Overnight requests were followed up retrospectively the following morning.

Categories were defined as follows:

(1) Acute surgical admission: a test used to assist in the decision to admit a patient to a surgical ward. 
INSTITUTE OF BIOCHEMISTRY : CLINICAL SERVICES GROUP

APPROPRIATENESS REVIEW (EMERGENCIES) : DRAFT STUDY FORM

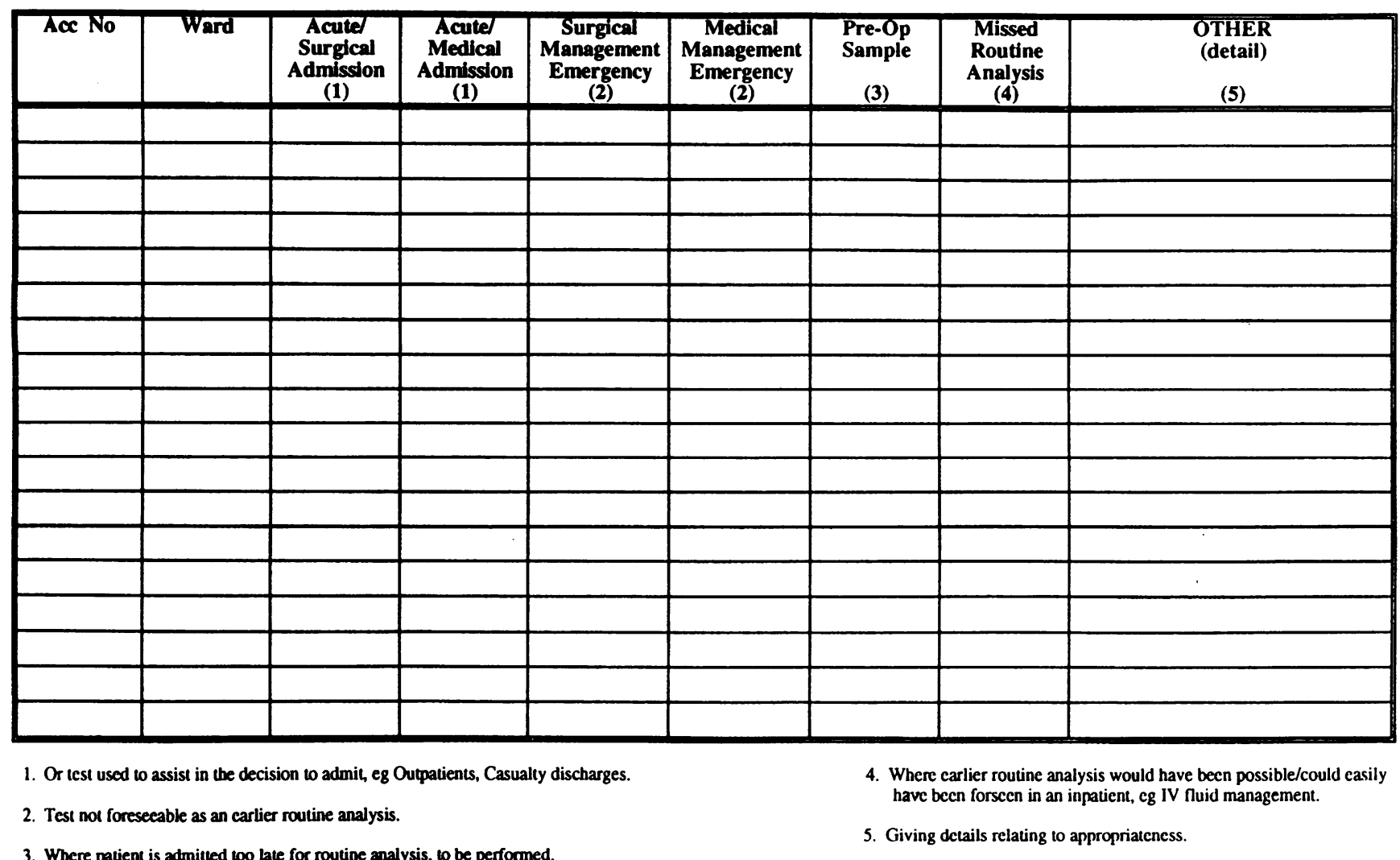

Appropriateness study form used to collect data on emergency requests over each 24 hour period.

(2) Acute medical admission: a test used to assist in the decision to admit a patient to a medical ward.

(3) Surgical management emergency: a test on a surgical inpatient, not foreseeable as an earlier routine analysis, due to a change in the patient's circumstances.

(4) Medical management emergency: a test on a medical inpatient, not foreseeable as an earlier routine analysis, due to a change in the patient's circumstances.

(5) Preoperative sample: a test on an elective admission for surgery taken too late for routine analysis to be performed prior to surgery.

(6) Missed routine analysis: a test performed where an earlier routine analysis should have been possible or could easily have been foreseen in a stable inpatient - for example, intravenous fluid management.

(7) Accelerated routine: a test on a stable inpatient sent before 1000 hours in the working day (subtracted retrospectively from the "others" category).

(8) "Others", including details relating to appropriateness (see later).

Using the criteria outlined above for appropriate use of an emergency biochemistry service, categories 1 to 4 were recorded as appropriate and categories 5 to 7 as inappropriate. Category 8 requests were allocated to the appropriate or inappropriate totals depending on the individual circumstances, according to the consensus view of the monitors.

No attempt was made to evaluate clinical judgement of the need for a test within these categories. Nor was any attempt made during the periods under review to influence requesting patterns or to discourage actively test requests other than through the normal departmental practice of daytime vetting of requests by the duty biochemist, and, out of hours, arrangement of specimens with the on call MLSO, with cover from on call medical staff.

EVALUATION OF CONCORDANCE

Prior to the study, all five monitors allocated a batch of 50 consecutive emergency forms to the categories shown in the figure, based purely on information provided on the request form. The categories listed by each of the monitors were then compared to evaluate individual differences in categorisation, with particular emphasis on appropriate and inappropriate categories. Results of this preliminary study were discussed jointly prior to the main study in order to improve consistency of categorisation.

\section{Results}

PRELIMINARY STUDY

Monitors were almost entirely concordant in assigning requests to the appropriate and inappropriate categories. At least four of five agreed on the overall appropriateness categorisation in all 50 requests, and no one monitor disagreed on more than one of the 50 tests. A total of four disagreements with the consensus view of appropriateness category was 
Table 1 Distribution of 509 emergency requests by five monitors into eight functional categories

\begin{tabular}{|c|c|c|c|c|c|c|c|c|c|c|}
\hline \multirow[b]{2}{*}{ Monitor } & \multicolumn{10}{|l|}{ Category } \\
\hline & $\begin{array}{l}\text { Acute } \\
\text { surgical } \\
\text { admission }\end{array}$ & $\begin{array}{l}\text { Acute } \\
\text { medical } \\
\text { admission }\end{array}$ & $\begin{array}{l}\text { Surgical } \\
\text { management } \\
\text { emergency }\end{array}$ & $\begin{array}{l}\text { Medical } \\
\text { management } \\
\text { emergency }\end{array}$ & $\begin{array}{l}\text { Total } \\
\text { appropriate } \\
\text { (including } \\
\text { "others") }\end{array}$ & $\begin{array}{l}\text { Pre- } \\
\text { operative } \\
\text { sample }\end{array}$ & $\begin{array}{l}\text { Missed } \\
\text { routine } \\
\text { analysis }\end{array}$ & $\begin{array}{l}\text { Accelerated } \\
\text { routine } \\
\text { analysis }\end{array}$ & "Others" & $\begin{array}{l}\text { Total } \\
\text { inappropriate } \\
\text { including } \\
\text { "others" }\end{array}$ \\
\hline $\begin{array}{l}1 \\
2 \\
3 \\
4 \\
5 \\
\text { Total }\end{array}$ & $\begin{array}{l}23(2) \\
21(2) \\
24(2) \\
15(2) \\
10(4) \\
93(2)\end{array}$ & $\begin{array}{r}26(1) \\
39(1) \\
36(1) \\
28(1) \\
33(1) \\
162(1)\end{array}$ & $\begin{array}{r}5(6) \\
10(5) \\
6(6) \\
9(5) \\
9(5) \\
39(5)\end{array}$ & $\begin{array}{l}18(3) \\
17(3) \\
14(3) \\
13(3) \\
26(2) \\
88(3)\end{array}$ & $\begin{array}{r}72 \\
87 \\
80 \\
66 \\
79 \\
384\end{array}$ & $\begin{array}{r}17(4) \\
14(4) \\
9(4) \\
10(4) \\
4(6) \\
54(4)\end{array}$ & $\begin{array}{r}6(5) \\
6(6) \\
9(4) \\
6(6) \\
15(3) \\
42(6)\end{array}$ & $\begin{array}{r}4(7) \\
6(6) \\
3(7) \\
3(7) \\
4(6) \\
20(7)\end{array}$ & $\begin{array}{l}3(8) \\
1(8) \\
2(8) \\
2(8) \\
1(8) \\
9(8)\end{array}$ & $\begin{array}{r}30 \\
27 \\
23 \\
21 \\
24 \\
125\end{array}$ \\
\hline
\end{tabular}

Categories 1-4 are combined in the appropriate total and categories 5-7 in the inappropriate total. The nine other requests have been categorised as either inappropriate $(n=7)$ or appropriate $(n=2)$ and added to the relevant total. The category rank by frequency is shown in brackets for each monitor-for example, all five monitors found acute medical admission to be the most frequent reason for a request.

Table 2 Percentage distribution of inappropriate requests by category. Each category is shown as a percentage of the number of inappropriate requests and as a percentage of the total number of emergency requests recorded

\begin{tabular}{lrlc}
\hline Request category & $n$ & Percentage of inappropriate & Percentage of total \\
\hline Preoperative & 54 & $43 \cdot 2$ & $10 \cdot 6$ \\
Missed routine & 42 & 33.6 & 8.3 \\
Accelerated routine & 20 & 16.0 & 3.9 \\
"Others" (for example, repeated & 9 & $7 \cdot 2$ & 1.8 \\
sample) & & & \\
\hline
\end{tabular}

found in 250 records examined. All classifications into medical and surgical categories were agreed between the five monitors. However, classification of newly admitted patients as acute admissions or inpatient management emergencies was a regular source of discordant categorisation. This distinction was anticipated and had no implications for overall appropriateness classification.

\section{MAIN STUDY}

A total of 509 requests was received during the five 24 hour periods. The distribution of requests by category is shown in table 1 . The "others" category consisted of six unintentional duplicates of samples which had already been sent (inappropriate), one sent in error to the emergency laboratory (inappropriate), and two samples sent during routine working hours because of laboratory problems with an earlier sample (breakage, lost sample) (appropriate). Other requests originally included in this category emerged after retrospective analysis as a separate category (category 7 above). Allocation of individual requests in the "others" category to either appropriate or inappropriate groups permitted a full breakdown of inappropriate requests (table 2 ). When the minor categories were grouped into the major appropriate and inappropriate categories, we found $75 \%(384 / 509)$ to be appropriate and $25 \%(125 / 509)$ to be inappropriate by the criteria used to define these categories.

The time distribution of requests received throughout the five periods was examined. Taken in conjunction with the breakdown of inappropriate requests (table 2), this demonstrates that the major inappropriate use of the service was attributable to requests sent as emergencies for administrative reasons (other than those relating to acute admissions), and followed a bimodal distribution, with a major peak before lunch time and a minor peak in the evening. Significantly, the great majority of evening and overnight requests were appropriate: only 25 of 125 inappropriate requests were received after 1700 hours, 12 of these after 2100 hours. Of these 25 , six were preoperative samples, eight were sent because of missed routine analysis, six were in the accelerated routine category, and five fell into the "others" category.

Concordance between the monitors was reconfirmed in the main study by jointly reviewing categorisation. In addition, the different categories were ranked by frequency by each monitor. Although strict comparison between monitors was not possible as each covered a different 24 hour period, the finding that each ranked the different categories in a similar order is consistent with concordant categorisation, and also suggests that test requesting patterns did not vary greatly from one 24 hour period to another.

\section{Discussion}

This study examined a specific aspect of appropriateness of medical care, namely the use of emergency tests within a biochemistry laboratory. We have used explicit criteria for appropriate use of an emergency biochemistry laboratory and have categorised requests accordingly. In a study of this kind, the term inappropriate is used for requests which fall outside the criteria used. It is essential to recognise that use of this term is not a reflection of poor clinical practice. For example, a number of emergency requests were categorised as inappropriate on the basis that an earlier routine request should have been possible. This study did not seek to establish whether such requests were due to oversight or whether they resulted from situations which made it impossible for the clinician to send a sample in time for routine analysis - for example, inpatient management emergency, ward round.

While, undoubtedly, a number of requests to a biochemistry emergency laboratory originate from minor errors such as forgetting to send a routine sample on an inpatient, it may be more useful to focus on situations where the request has arisen as a result of routine practice-for example, accelerated routine (category 7 ). From an examination of these inappropriate categories (categories 5 to 7 ) we have identified three strategies to improve local targeting of resources. 
Firstly, whilst surgical colleagues may be encouraged to admit their patients early in the day prior to surgery, this may not be administratively possible in a busy hospital. The problem of preoperative samples may be tackled more successfully by examining hospital facilities for surgical patients with a view to providing a service where routine administrative and laboratory needs may be addressed without admitting the patient to hospital (for example, an outpatient triage centre).

Secondly, the group of tests sent too late for routine analysis may be addressed by discussion with clinical colleagues to identify what proportion of these tests results from the timing of ward rounds and specimen collection times, and by re-examining the phlebotomy services (where they exist) and/or collection times for the wards concerned.

Thirdly, the problem of requests which were categorised as accelerated routine may be addressed by examining the existing facilities in the laboratory for prioritising urgent routine samples.

An emergency laboratory will always receive a number of inappropriate requests. The decision to target resources towards changing laboratory or clinical practice in order to reduce this number must be based both on the existing capacity available within an emergency laboratory to deal with additional inappropriate tests and the ease with which such requests may be redirected towards other parts of the laboratory service. In this study we identified $25 \%$ of all emergency requests which did not fulfil our criteria for appropriateness. This raises the issue of who decides what is appropriate. We have made no attempt in this study to pass judgement on clinical decision-making in individual cases. The issue of clinical relevance is quite separate and cannot be assessed purely from a laboratory standpoint. The categories used in this study were chosen to reflect accurately working practice within the hospital, and were defined as objectively as possible with a view to their practical application. Where the terms of definition have been inherently subjective we have attempted to minimise arbitrary application by conducting peer review before and during the main study.

What are the practical implications of these findings? Implementation of these strategies in our hospital is currently underway. Some changes to hospital practice may be achieved at minimal additional cost, whereas others require more substantial resources. These will only be made available if a case based on accurate and credible data can be made to hospital management, in conjunction with ward based clinical colleagues. This applies especially to the provision of an outpatient service to meet routine administrative and laboratory needs. Our findings have been used in ongoing consultations with hospital colleagues, the aim of which is to provide such a service. Phlebotomy and portering services are more amenable to restructuring, and both bleeding and collection times have been changed in some wards to facilitate the provision of a more efficient emergency biochemistry service. In addition, we have reviewed our internal laboratory system for prioritising urgent routine samples and in certain instances have redirected incoming requests. Full implementation of the strategies identified in this preliminary study will permit closure of the audit loop by repeating the evaluation.

We are grateful to Miss P Price for her help in preparing this manuscript.

1 Brook RH. Appropriateness: the next frontier. BMF 1994; 308:218-19.

2 Bluth EI, Lambert DJ, Lohmann TP, Franklin DN, Bourgeois $\mathrm{RN}$, Kardinal CG, et al. Improvement in stat laboratory turnround time, a model continuous quality improvement project. Arch Intern Med 1992;152:837-40. 